Letter to the Editor

\section{Neuromyelitis optica spectrum disorder and varicella-zoster infection}

Keywords:

Varicella-zoster virus

Neuromyelitis optica spectrum disorder

\section{Background}

Neuromyelitis Optica (NMO) is an autoimmune condition that predominantly targets optic nerves and spinal cord. The discovery of NMO-IgG and its target aquaporin-4 (AQP4) as a marker of NMO allowed a better understanding of the disease and, recently, a new definition and diagnostic criteria for NMO spectrum diseases (NMOSD) have been proposed [1]. In a subgroup of patients with NMOSD, a parainfectious pathogenesis may play a central role [2].

\section{Case report}

A 77-year-old woman was admitted for a vesicular erythematous rash in left L4-S1 dermatomes followed 2 days later by rapidly progressive paraparesis, sensory level by L4 and urine retention. Vision was not impaired. She presented a medical history of essential tremor and autoimmune thyroiditis and was medicated with clopidogrel, levothyroxine and zolpidem. Spinal cord MRI demonstrated a longitudinal, T2-hyperintense lesion extending from C2-C3 to Th12 with no gadolinium enhancement (Fig. 1-A, B). Cerebrospinal fluid (CSF) analysis revealed lymphocytic pleocytosis (107 cells/ $\mu \mathrm{l} ; 70 \%$ lymphocytes), increased total protein $(1.87 \mathrm{~g} / \mathrm{L})$ and positive PCR for Varicella Zoster Virus (VZV) DNA. An extensive VZV meningomyelo-radiculitis was diagnosed and she was treated for 21 days with acyclovir, weaning scheme of steroids and rehabilitation. At discharge, she had a grade 2 paraparesis, and during follow-up she remained wheelchairbound and had a chronic indwelling urinary catheter.

Six months later, there was an acute progressive development of involuntary movements of the upper limbs with worsening in the week before admission. Neurological examination showed cerebellar dysarthria, bilateral finger-nose dysmetria and choreiform movements (right side predominant). Brain MRI revealed T2-hyperintense lesions located in the periependimal surface of the fourth ventricle, extending to the right dentate nucleus and bilateral middle cerebellar peduncles (Fig. 1-C, D). Complete basic and immunological analyses were normal, except positive anti-SSA and SSB and persistently elevated sedimentation rate. CSF showed increased total protein $(0.50 \mathrm{~g} / \mathrm{L})$, no cells and negative PCR for viruses. Unmatched oligoclonal bands were found in CSF (intratecal synthesis). The patient was medicated with gabapentin and clonazepam, with a slight improvement in choreiform movements. She started immunosuppression with prednisolone ( $60 \mathrm{mg} /$ day) and azathioprine (100 mg/day) on suspicion of inflammatory or autoimmune CNS disease. Review of systemic symptoms disclosed xerostomy and Schirmer test confirmed reduced lacrimation. The patient denied ocular complaints and, at that time, she was being treated with several drugs that can cause xerostomy; therefore, it was decided not to perform further investigation. Anti-AQP4 in serum collected during the second episode was later found to be positive in indirect immunofluorescence serum assay (tested only for positivity with 1:10 dilution). The acute brainstem syndrome, anti-AQP4 seropositivity and NMOSDtypical brain lesion pattern are diagnostic for NMOSD.

\section{Discussion}

The differential diagnosis of longitudinally extensive transverse myelitis is vast, but in our patient an infectious etiology by VZV was confirmed. The appearance of new neurological symptoms and cerebellar lesions 6 months after infectious myelitis led to further etiologic investigation. The analytical results of the blood and CSF generated the suspicion of an auto-immune or inflammatory disease. Sjögren syndrome (SS) was considered as a possible cause for the recurrent neurological manifestations and we cannot definitely exclude this diagnosis. Despite the presence of clinical and laboratorial findings suggestive of SS and unmatched oligoclonal bands (which occur in $20 \%$ of patients with NMOSD), the typical neuroimaging findings and anti-AQP4 seropositivity confirmed NMOSD. Recently an international consensus on NMOSD suggested that co-occurrence of clinical symptoms of SS, NMOSD clinical syndrome and positive anti-AQP4 strengthens confidence on a NMOSD diagnosis [1]. The underlying cause of CNS manifestations is more likely to be caused by NMOSD than a complication of SS $[3,4]$.

Reviewing the literature, we found nine cases of NMOSD in association with VZV infection [2,4-6]. All patients were female and full recovery occurred in 3/9 patients. The main differences of our patient when compared to the previously reported cases are: older age, longer time interval between VZV infection and occurrence of neurological symptoms attributable to NMOSD and positive PCR for VZV DNA in CSF.

Triggering role of infectious events in the development of NMOSD was suggested. Sellner and colleagues reported that antecedent viral infections account for 15-35\% in NMOSD and there is a predominant association with VZV [2]. Other viral infections associated with NMOSD have been reported and include human immunodeficiency virus, cytomegalovirus, Dengue virus, Hepatitis A and Epstein-Barr Virus [2,7]. Mechanisms often used to explain the association of autoimmunity and virus infection are molecular mimicry and bystander activation. Bystander activation involves inflammatory reaction and tissue destruction which may result in release of AQP4 from the nervous system, followed by antibody production. Molecular mimicry relies on antigenic similarity between structural epitopes or peptide sequences of an infectious agent and host-proteins. Currently available data is not sufficient to confirm the pathogenic 

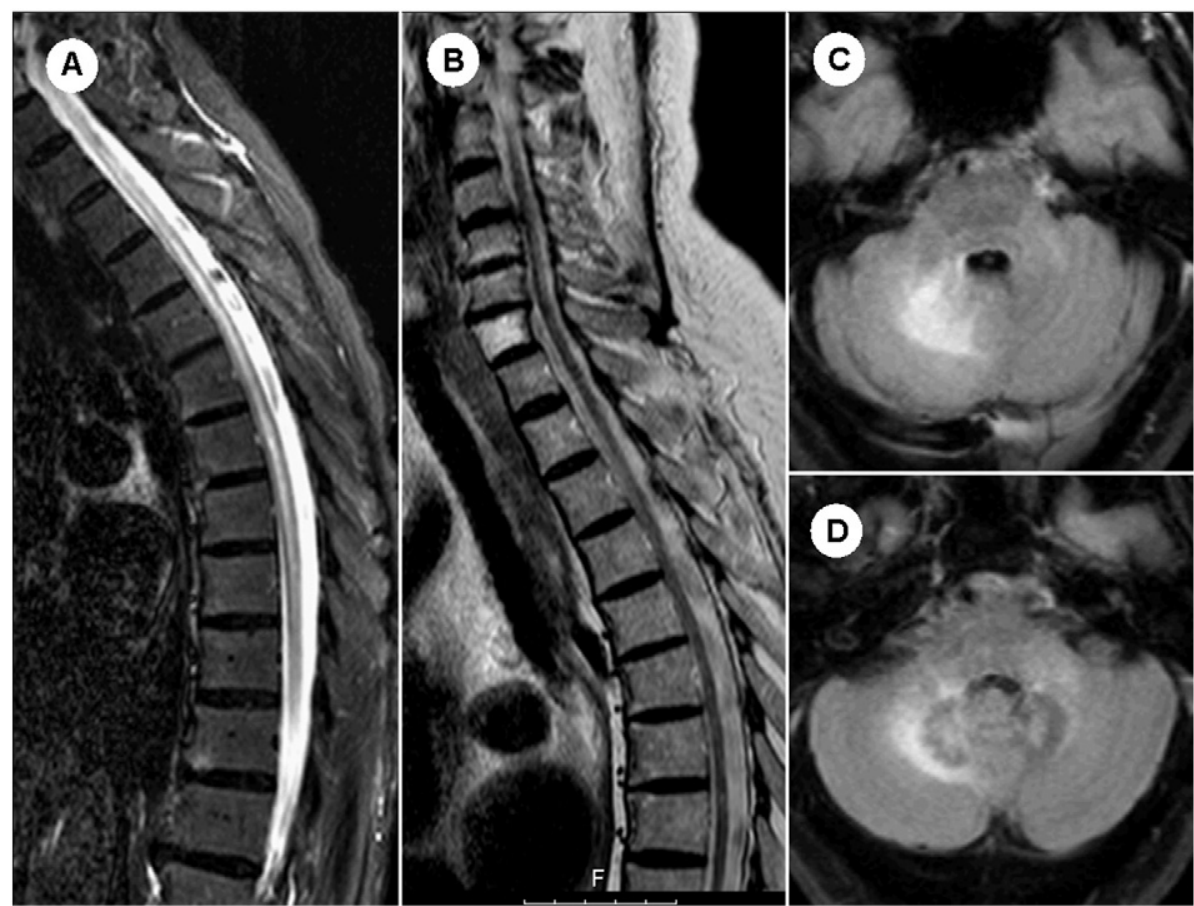

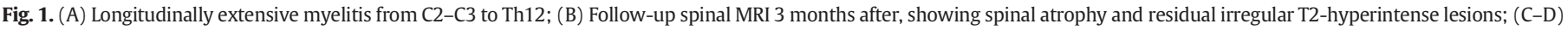
T2-hyperintense lesions located on right dentate nucleus and bilateral middle cerebellar peduncles in brain MRI performed 19 months after VZV meningo-myelo-radiculitis.

relevance of previous infections in the pathogenesis of NMOSD, as suggested by the temporal proximity $[2,8]$.

This is the first report of a patient presenting a non-opticospinal phenotype of NMOSD after VZV infection. The diagnosis of NMOSD might have been suspected when the first episode of VZV myelopathy occurred, based on the considerable longitudinal extent of the myelopathy. We hypothesize that blood-brain barrier breakdown during VZV myelitis triggered the production of anti-AQP4 and allowed access to the central nervous system, which contributed to the severity of the initial infectious myelitis and generated the possibility of recurrent NMOSD events.

\section{References}

[1] D.M. Wingerchuk, B. Banwell, J.L. Bennett, et al., International consensus diagnostic criteria for neuromyelitis optica spectrum disease, Neurology 85 (2015) 177-189.

[2] J. Sellner, B. Hemmer, M. Mühlau, The clinical spectrum and immunobiology of parainfectious neuromyelitis optica (Devic) syndromes, J. Autoimmun. 34 (4) (2010) 371-379.

[3] S. Jarius, C. Jacobi, J. de Seze, et al., Frequency and syndrome specificity of antibodies to aquaporin-4 in neurological patients with rheumatic disorders, Mult. Scler. 17 (2011) 1067-1073.

[4] A. Jayarangaiah, R. Sehgal, N. Epperla, Sjögren's syndrome and neuromyelitis optica spectrum disorders (NMOSD) - a case report and review of the literature, BMC Neurol. 14 (2014) 200

[5] J.S. Park, S.J. Hwang, J.H. Shin, et al., A recurrent longitudinally extensive transverse myelitis with Aquaporin-4(AQP4) antibody after herpes zoster, J. Neurol. Sci. 334 (2013) 69-71.

[6] K. Heerlein, S. Jarius, C. Jacobi, et al., Aquaporin-4 antibody positive longitudinally extensive transverse myelitis following varicella zoster infection, J. Neurol. Sci. 276 (2009) 184-186.
[7] S. Yoshimura, N. Isobe, T. Matsushita, et al., Distinct genetic and infectious profiles in Japanese neuromyelitis optica patients according to anti-aquaporin 4 antibody status, J. Neurol. Neurosurg. Psychiatry 84 (2013) 129-134.

[8] J. Sellner, S. Cepok, S.R. Kalluri, et al., Aquaporin 4 antibody positive central nervous system autoimmunity and multiple sclerosis are characterized by a distinct profile of antibodies to herpes viruses, Neurochem. Int. 57 (2010) 662-667.

Célia Machado

Neurology Department, Hospital de Braga, Portugal Corresponding author at: Sete Fontes, São Victor, 4710-243 Braga, Portugal. E-mail address: ccarinamachado@gmail.com.

José Amorim Jaime Rocha

Neurology Department, Hospital de Braga, Portugal E-mail addresses: josemvamorim@gmail.com (J. Amorim); jaimeroc@gmail.com (J. Rocha).

João Pereira Esmeralda Lourenço João Pinho

Neurology Department, Hospital de Braga, Portugal E-mail addresses: joaocampospereira@sapo.pt (J. Pereira); esmeraldalourenco@gmail.com (E. Lourenço); jdpinho@gmail.com (J. Pinho).

26 August 2015 